\title{
The Geometry of Gauge Field Copies ${ }^{\star}$
}

\author{
Francisco Antonio Doria ${ }^{\star \star}$ \\ Department of Mathematics, University of Rochester, Rochester, NY 14627, USA
}

To the Memory of Jorge André Swieca

\begin{abstract}
We show that a gauge field uniquely determines its potential if and only if its holonomy group coincides with the gauge group on every open set in spacetime, provided that the field is not degenerate as a 2-form over spacetime. In other words, there is no potential ambiguity whenever such a field is irreducible everywhere in spacetime. We then show that the ambiguous potentials for those gauge fields are partitioned into gauge-equivalence classes (modulo certain homotopy classes) as a consequence of the nontrivial connectivity of spacetime. These homotopy classes depend on the gauge group, on the holonomy group and on this last group's centralizer in the gauge group.
\end{abstract}

\section{Introduction}

The potential ambiguity for nonabelian gauge fields, that is, the existence of "gauge field copies", has been widely discussed [1] since it was discovered by Wu and Yang [2]. Some gauge fields admit two or more potentials. This potential ambiguity falls into two large classes with a nonvoid intersection: ambiguous potentials are sometimes (but not always) equivalent modulo a gauge transformation. There is also an in-between situation, for some gauge fields admit at each gauge a whole system of potentials which is partly but not completely reducible with the help of gauge mappings [3, 37].

The present paper gives necessary and sufficient conditions for the existence or inexistence of potential ambiguities in a given gauge field provided that the gauge field is not degenerate as a 2 -form on spacetime. Potential ambiguity is shown here to be a consequence of too many degrees of freedom in the field. In other words, if we adopt the bundle description [4] for gauge fields, there will be a potential ambiguity whenever the bundle is (at least locally) reducible. When the bundle is maximally twisted, that is, when the holonomy group coincides with the whole gauge group on every open set in the base manifold, we find no copies at all.

$\star \quad$ Research supported by C.N.Pq. and M.E.C. (Brazil)

$\star \star$ Current address: Instituto de Fisica, Universidade Federal do Rio de Janeiro, BR-21910 R1o de Janeiro RJ, Brazil 


\section{The Geometry of Gauge Fields}

We give here a self-contained description of classical gauge field theory as a theory of connections on a principal fiber bundle. Some proofs are omitted and referred to the literature.

\section{a) The Overall Setting}

Let $M$ be a 4-dimensional Hausdorff real connected and complete smooth manifold (in what follows we will sometimes write "smooth" for " $C^{\infty}$ "). $M$ is to be seen as a spacetime without a fixed metric tensor; in Sect. 3 of this paper we will use an arbitrary non-degenerate smooth metric tensor to simplify the discussion of some expressions. The particular metric used has no bearing on our results, as we show below.

Let $G$ be a finite-dimensional semi-simple Lie group which will be identified with one of its faithful matrix representations; $G$ will be called the gauge group. Its Lie algebra will be denoted $L(G)$.

We will consider all principal fiber bundles $[5] P(M, G)_{\lambda}$, where $\lambda$ denotes the isomorphism class to which the bundle belongs. We will also write $P$ for the bundle's total space. $M, G$, and $P$ are in the most general situation finitedimensional and noncompact. Compact $M$ appears in the standard formulation of Euclidean gauge theories [6] and when we deal with zero-mass, conformallyinvariant fields [7]. Compact $G$ is a common assumption in most gauge theories. If $M$ and $G$ are compact, so is $P$. All our results are however independent of compactness assumptions.

For the following summary of geometrical notions see [8].

Let $T . P$ be the tangent bundle of $P$. We define:

2.1. Definition. A connection on $P$ is a smooth decomposition T. $P=\mathscr{H} \oplus \mathscr{V}$ which satisfies: (I) Given the principal bundle projection $\pi: P \mapsto M, d \pi: \mathscr{H} \mapsto d \pi(\mathscr{H})$ $=T . M$, where $T . M$ is the tangent bundle of $M$. (II) For $p \in P, a \in G$, the map $p \mapsto p a$ implies

$$
\mathscr{H}|p \mapsto \mathscr{H}| p a=R_{a}^{*}(\mathscr{H} \mid p),
$$

where the $\mid$ denotes a restriction to the point and $R_{a}^{*}$ denotes the induced right action.

A connection form $\alpha$ on $P$ is a linear map $\alpha: T . P \mapsto L(G)$ given by the conditions

$$
\begin{aligned}
\alpha(\mathscr{H}) & =0 . \\
R_{a}^{*} \alpha & =\operatorname{Ad}\left(a^{-1}\right)(\alpha),
\end{aligned}
$$

where Ad denotes the induced group action $G \times L(G) \mapsto L(G)$ on its Lie algebra as a representation space.

2.2. Remark. The connection and connection form are always taken to be continuous and differentiable all over $P$.

Let $X_{1}, X_{2}: P \mapsto T . P$ be smooth vectorfields on the bundle $P$. Let us also be given the exterior bundle of $P$ endowed with the usual exterior derivative. If $\eta$ denotes the projection

one has:

$$
\eta: T . P \mapsto \mathscr{H},
$$


2.3. Definition. If $\alpha$ is the connection form, the curvature form $\varphi$ w.r.t. $\alpha$ on $P$ is given by

$$
\varphi\left(X_{1}, X_{2}\right)=D \alpha\left(X_{1}, X_{2}\right) \underset{\text { def }}{=} d \alpha\left(\eta X_{1}, \eta X_{2}\right) .
$$

The Cartan structure equations are [9]:

2.4. Lemma.

$$
\begin{gathered}
\varphi\left(X_{1}, X_{2}\right)=d \alpha\left(X_{1}, X_{2}\right)+\frac{1}{2}\left[\alpha\left(X_{1}\right), \alpha\left(X_{2}\right)\right]_{-} \\
D \varphi\left(X_{1}, X_{2}, X_{3}\right) \underset{\operatorname{def}}{=} d \varphi\left(\eta X_{1}, \eta X_{2}, \eta X_{3}\right)=0
\end{gathered}
$$

b) Gauge Fields and Potentials: Local Description

We are now going to relate geometry and physics, that is, we are going to interprete these structures as gauge fields and gauge potentials. Let $P$ be a principal bundle as described above. If $P$ is nontrivial, it will admit only local cross-sections. Let us be given $\mathscr{U}$, a maximally trivializing open covering for $P$, that is,

$$
\mathscr{U}=\{U \subseteq M \mid M=\bigcup U\},
$$

and for any two sets that meet, $U_{1}$ and $U_{2}$ in $\mathscr{U}$, the bundle $P \mid U_{1} \bigcup U_{2}$ is not trivial, while $P \mid U_{i}=U_{i} \times G, i=1,2$. Let us be given a family of $\mathscr{U}$-dependent local crosssections

$$
\sigma_{U}: U \mapsto P_{U},
$$

for $U \in \mathscr{U}$ and $P_{U}=P \mid U$. For the overlappings $U \cap U^{\prime} \neq \emptyset$, let us have the mappings

$$
\tau_{U U^{\prime}}: \sigma_{U^{\prime}} \mapsto \sigma_{U^{\prime}}=\sigma_{U} \tau_{U U^{\prime}} \mid U \cap U^{\prime},
$$

where the

$$
\tau_{U U^{\prime}}: U \cap U^{\prime} \mapsto G .
$$

2.5. Definition. A gauge

$$
\sigma: M \mapsto P
$$

is a triple

$$
\sigma=\left\{\mathscr{U} \cdot \sigma_{U}, \tau_{U U^{\prime}}\right\}
$$

such that for all nonvoid overlappings $U \cap U^{\prime}$ we identify

$$
\sigma_{U}\left|U \cap U^{\prime} \equiv \sigma_{U^{\prime}}\right| U \cap U^{\prime}\left(\bmod \tau_{U U^{\prime}}\right) .
$$

2.6. Remark. The maps $\tau_{U U^{\prime}}$ are called local gauge transformations. The global gauge transformations are defined below, and related to these local transformations.

Let $X_{d}: M \mapsto T . M$ be a vectorfield on $M$ ( $d$ stands for "downstairs"). Given a gauge $\sigma$, consider the induced mapping

$$
d \sigma: T . M \mapsto T . \sigma(M)
$$


given by

$$
X_{d} \mapsto d \sigma\left(X_{d}\right) \underset{\mathrm{def}}{=} X_{d}^{(\sigma)} .
$$

2.7. Remark. We notice in particular that, for $p \in \sigma(x)$ such that $\pi(p)=x \in M$, there is an isomorphism

$$
\iota: T . \sigma(M)|p \mapsto \mathscr{H}| p
$$

given by

$$
l=(d \pi)^{-1} \circ(d \sigma)^{-1} .
$$

That is, at each point, the tangent space to the gauge and the horizontal subspace are isomorphic (but not necessarily identical).

Gauge fields and gauge potentials are:

2.8. Definition. (I) The gauge potential $A^{(\sigma)}\left(X_{d}\right)$ at the gauge $\sigma$ is given by the restriction to $\sigma$ of the connection form $\alpha$ as follows :

$$
A^{(\sigma)}\left(X_{d}\right) \underset{\operatorname{def}}{=} \alpha\left(X_{d}^{(\sigma)}\right) .
$$

(II) The gauge field $F^{(\sigma)}\left(X_{d}, Y_{d}\right)$ at the gauge $\sigma$ is defined by the restriction to $\sigma$ of the curvature form (2.1) as follows :

$$
F^{(\sigma)}\left(X_{d}, Y_{d}\right) \underset{\operatorname{def}}{=} 2 \varphi\left(X_{d}^{(\sigma)}, Y_{d}^{(\sigma)}\right) .
$$

2.9. Remark. If one takes a coordinate system $\left(x^{\mu}\right)$ at $U \subset M$, such that the bundle is trivial over $U$, given the local holonomous reference system at $U$ for the tangent space, $\left[\partial_{\mu}\right]$,

$$
\partial_{\mu} \equiv \partial / \partial x^{\mu}
$$

and if we consider a constant ("flat") local cross-section $(x, 1)$, where 1 is the group's identity element, for this particular local cross-section $\sigma$ one has:

$$
F_{\mu \nu}=\partial_{\mu} A_{\nu}-\partial_{\nu} A_{\mu}+\left[A_{\mu}, A_{\nu}\right]_{-},
$$

with

$$
\begin{gathered}
A_{\mu}=A^{(\sigma)}\left(\partial_{\mu}\right) \\
F_{\mu \nu}=F^{(\sigma)}\left(\partial_{\mu}, \partial_{\nu}\right) .
\end{gathered}
$$

The connection and curvature forms are continuous on $P$, even if the bundle is nontrivial. However this is not in general true of the gauge potential and field, which may need several coordinate patches and nontrivial local gauge transformations to be described on the whole of $M$. The chief example of this situation is the $\mathrm{Wu}$ and Yang magnetic monopole [10]. This gauge-dependent picture has actually been first proposed by those two authors. While providing a straightforward local geometrical picture for the physical concepts, it may lead to trouble in the global situation: consider for instance the nontrivial $\mathbb{Z}_{3}$ principal bundle over the circle $S^{1}$. One can get two gauges in this bundle, with respect to the same partition of the base space $S^{1}$ into two overlapping half-circles which are not topologically equivalent. The restriction of the same bundle-defined object to these 
two nonequivalent gauges cannot be made equivalent modulo a global gauge transformation (see below for its definition). This example is due to John Harper [11].

\section{c) Gauge Fields as Tensorial Forms}

An alternative description for gauge fields can be provided with the help of the equivalence between "tensorial forms" on the bundle and exterior forms over the base space with values in a given vector space [12]. Let us be given the principal bundle $P$ and its $V$-valued exterior bundle $\Omega_{V}(P)$, where $V$ is a representation space for the gauge group $G$. A cross-section

$$
\beta: P \mapsto \Omega_{V}(P)
$$

is called a tensorial form [13] if it is zero on vertical vectors (w.r.t. a given connection) over $P$. The curvature form (2.1) is a tensorial form; the connection form obviously not. Tensorial forms over $P$ are naturally mapped on $V$-valued forms over the basis manifold. Let $X^{h}, Y^{h}, \ldots$ be horizontal vectorfields on $P$, that is, fields for which

$$
\alpha\left(X^{h}\right)=0,
$$

where $\alpha$ is the connection form. Given the bundle projection

$$
\pi: P \mapsto M,
$$

we put

$$
X_{d}=d \pi\left(X^{h}\right)
$$

We then have:

2.10. Proposition. Let $p \in P$. If we define the gauge field $F$ on $M$ in a pointwise fashion by

$$
F\left(X_{d}, Y_{d}\right)\left|\pi(p)=p\left(2 \varphi\left(X^{h}, Y^{h}\right)\right) p^{-1}\right| p
$$

and for a cross-section $\xi$ of the bundle associated to $P$ with fiber $E$, induced by a fixed faithful representation of $G$ on $E$, the first structure Eq. (2.2) becomes

$$
F\left(X_{d}, Y_{d}\right) \xi=\left[D_{X_{d}}, D_{Y_{d}}\right]_{-} \xi-D_{\left[X_{d}, Y_{d}\right]_{-}} \xi
$$

and the second structure equation (the Bianchi differential identities) are

$$
D_{X_{d}} F\left(Y_{d}, Z_{d}\right)+D_{Z_{d}} F\left(X_{d}, Y_{d}\right)+D_{Y_{d}} F\left(X_{d}, Y_{d}\right)=0 \text {, }
$$

where the covariant derivative is defined by

$$
D_{X_{d}} \xi\left|\pi(p)=p X^{h} \xi\right| p \text {. }
$$

Proof. Same as in the Riemannian case (see for example Sternberg [14]).

In local coordinates $(2.12)$ can be written

$$
D_{\partial_{\mu}} \xi=D_{\mu} \xi=\partial_{\mu} \xi+A_{\mu} \xi
$$


for $X=\partial_{\mu}$ and $\xi$ with values in the lowest dimensional representation space for $G$. (2.10) is

$$
F\left(\partial_{\mu}, \partial_{v}\right)=F_{\mu \nu}=\partial_{\mu} A_{v}-\partial_{v} A_{\mu}+\left[A_{\mu}, A_{v}\right]_{-}
$$

and (2.12),

$$
D_{\mu} F_{v \varrho}+D_{\varrho} F_{\mu \nu}+D_{v} F_{\varrho \mu}=0 .
$$

2.11. Remark. In general a local description for the gauge field such as (2.14) can be only achieved with the help of several coordinate patches on the bundle. A gauge is a particular choice of such coordinate patches. The restriction of (2.1) to a gauge $\sigma$ gives us an equivalence between the gauge dependent picture described in Part $b$ ) of the present section and the tensorial form description in Proposition 2.10. The expression of $\varphi$ at each local constant ("flat") cross-section $\sigma_{U}$ in a particular gauge [like the one before (2.6)] can be understood as the value of (2.9) at a local coordinate system in the bundle.

The relationship between the $L(G)$-valued 1-form $A$ on $M$ and the connection form $\alpha$ on $P$ is given by

$$
\alpha(p)=p^{-1} d p+p^{-1} A^{*} p
$$

where $A^{*}$ is the lift of $A$ to the identity local cross-section $(U, 1)$ and $p \in P$, such that its projection lies in $U$ [15].

We conclude with:

2.12. Definition. (I) A gauge transformation is a smooth diffeomorphism $u: P \mapsto P$ of the principal bundle which satisfies (A) for every $p \in P$ there exists $g(p) \in G$ such that $u(p)=p g(p)$; (B) $g(p a)=a^{-1} g(p) a$, where $a \in G$. (II) A local gauge transformation over $U \subseteq M$ is a gauge transformation of the bundle $P_{U}$.

The group of gauge transformations $\mathscr{G}$ is the group of all maps such as the one defined in (I). The group of all local gauge transformations over $U, \mathscr{G}_{U}$ is similarly defined. For details see Daniel and Viallet [16].

2.13. Remark. (I) A gauge transformation is equivariant, that is, $u(p a)=u(p) a$. It also induces the identity map on the basis manifold. (II) If $F^{(\sigma)}$ is a gauge field at the gauge $\sigma$, the action of $u$ on $F^{(\sigma)}$ is given by

$$
u\left(F^{(\sigma)}\right)=\operatorname{Ad}\left(u^{-1}\right) F^{(\sigma)}=u^{-1} F^{(\sigma)} u .
$$

If $A^{(\sigma)}$ is a gauge potential at the gauge $\sigma$, the action of $u$ on $A^{(\sigma)}$ is given by

$$
u\left(A^{(\sigma)}\right)=A^{(\sigma)}+u^{-1}\left(D\left(A^{(\sigma)}\right)\right) u
$$

where

$$
D\left(A^{(\sigma)}\right) u=d u+\left[A^{(\sigma)}, u\right]_{-} .
$$

(III) The objects $\tau$ in Definition 2.5 can be seen as local gauge transformations over $U \cap U^{\prime}$ acting on the local cross-section $\sigma_{U}$. The gluing together of local crosssections to form a gauge can be thus seen as the action of local gauge transformations at overlapping coordinate patches. (IV) As a consequence of the equivalence between the gauge picture and the tensorial-form picture, the 
connection form can be locally described by a Lie-algebra valued 1-form over a trivializing domain $U \subset M$ for the bundle. Both are smooth but require in general several patches for a coordinate description.

Now let $H \subset G$ be a Lie subgroup, and let $Q(M, H)$ be a principal fiber bundle with $H$ as its fiber.

2.14. Definition. Let $Q, P$ have the same basis manifold. (I) A smooth map $r: Q \mapsto P$ is a reduction of the structure group of the bundle $P$ if:

(A) $r: Q \mapsto P$ is a manifold embedding.

(B) $r$ induces the identity map on the basis manifold.

(C) For $p \in Q, a \in H, r(p a)=r(p) f(a)$, where $f: H \mapsto G$ is a monomorphism.

(II) For a nonvoid $U \subset M$,

$$
r_{U}: Q_{U} \mapsto P_{U}
$$

is a local reduction over $U$ of the bundle $P$ if it is a reduction of the bundle $P_{U}$.

\section{d) The Gauge Field Copy Problem}

For a summary of the best known examples and pathologies of the copy problem see Bollini et al. [17] and Deser and Drechsler [18]. The copy problem concerns the relationship between gauge potentials and gauge fields. It can be stated as follows :

(A) find necessary and sufficient conditions for the map between gauge fields and gauge potentials to be one-to-one;

(B) characterize in geometrical terms the ambiguous potentials;

(C) partition into gauge equivalence classes the ambiguous potentials.

There remains one last point: is the copy problem local? That is, can we find a field which is copied on $U$ and non-copied on $M-U$ ? The answer is yes:

2.15. Example. Given the trivial bundle $P=\mathbb{R}^{4} \times G$, where $G=\operatorname{SO}(3, \mathbb{R})$, let $E^{i}$, $i=1,2,3$ be the generators of the corresponding Lie algebra. Let $x=\left(x^{1}, x^{2}, x^{3}, x^{4}\right)$ be the standard Cartesian coordinates for $R^{4}$. Now consider two smooth functions :

(A) $f: \mathbb{R} \mapsto \mathbb{R}$, such that $f^{-1}(0)=[q, r] \subset \mathbb{R}$, a closed interval.

(B) $h: \mathbb{R} \mapsto \mathbb{R}$, such that $h(x)=0$ if $x \in \mathbb{R}-(q, r)$.

Define the potential $A(x)=A_{\mu}(x)$ in the local coordinate system:

$$
\begin{gathered}
A_{1}(x)=f\left(x^{1}\right)\left(E^{1}+\left(x^{2}\right) E^{2}\right), \\
A_{2}=A_{3}=A_{4}=0 .
\end{gathered}
$$

And $A^{\prime}(x)$ by

$$
\begin{gathered}
A_{1}^{\prime}(x)=A_{1}(x)+h\left(x^{1}\right) E^{1}, \\
A_{2}^{\prime}=A_{3}^{\prime}=A_{4}^{\prime}=0 .
\end{gathered}
$$

For both examples we have:

$$
F_{12}=-f\left(x^{1}\right) E^{2}
$$


while all other components are zero. We notice that for $x^{1} \in \mathbb{R}-(q, r)$ both potentials are coincident. They are also gauge-equivalent since one can easily check that the transformation given by

$$
u(x)=u\left(x^{1}\right)=\exp E^{1} \int_{0}^{1} h\left(x^{1} t\right) x^{1} d t
$$

is a (local) gauge transformation that sends $A^{\prime}$ over $A$ while keeping $F_{12}$ invariant. $19]$.

So the phenomenon is sometimes a local one. This example is due to Mostow

\section{The Main Result}

In this section we consider the gauge field $F$ to be a Lie algebra valued 2-form over $M$, as in Part c) of the preceding section. $F$ is always taken to be of class $C^{\infty}$ on $M$. Its potential, $A$, is a Lie algebra valued 1 -form of class $C^{\infty}$ as before; throughout this section we suppose that $F\left(X_{d}, Y_{d}\right)=0$ iff $X_{d}\left(\right.$ or $\left.Y_{d}\right)=0$, or $X_{d}=Y_{d}$ [25]. This will be used in the proof of Proposition 3.6 below.

The example at the end of Sect. 2 suggests the following definition as our starting point:

3.1. Definition. $F$ is said to have a local potential ambiguity on $M$ if there is a nonvoid $U \subseteq M$ such that $F \mid U$ is a field for at least two different potentials, $A$ and $B$, on $U$. If $U=M, F$ is said to have a global potential ambiguity.

3.2. Remarks. (I) It is easy to see that the action of the gauge transformation group $\mathscr{G}$ on $F$ sends copied fields over copied fields. (II) This definition includes the possibility of existence of a (local) gauge transformation that maps $A$ on $B$ while keeping $F$ invariant.

We now say that an object is defined almost everywhere on $M$ if it is defined on a subset $M_{0} \subset M$ which is (I) open and (II) dense in $M$. We then assert:

3.3. Lemma. $F$ has a single potential on $M$ if it is smooth on $M$ and has a single potential almost everywhere on $M$.

Proof. If $F$ has a single potential almost everywhere on $M$ and is smooth over the entire manifold, both the field and its potential can be uniquely and smoothly extended to $M$. The converse is immediate.

In what follows we remember that $G$ is supposed to be semi-simple. We also suppose that $F$ is a field for the potential $A$, associated to the connection form $\alpha$ on the bundle $P$. We will now consider the connection-endowed bundles $(P, \alpha)$.

3.4. Definition. (I) The field $F$ or the potential $A$ are said to be reducible if the corresponding connection-endowed bundle $(P, \alpha)$ is reducible. (II) $F$ (or $A$ ) are locally reducible over $U, U$ being a nonvoid open set in $M$, if $(P, \alpha) \mid U$ is reducible. (III) $F$ (or $A$ ) are said to be fully irreducible if they are not locally reducible.

We notice that local reducibility is a requirement which is weaker than reducibility. 
a) Necessary and Sufficient Conditions for the Existence of Copies

Our main result is :

3.5. Theorem. The following conditions are equivalent: (I) $F$ has a single potential on every open neighborhood in $M$; (II) $F$ is fully irreducible; (III) the centralizer in $G$ of $H_{F}, C_{G}\left(H_{F}\right)$, coincides with the center of $H_{F}$ on every open neighborhood in $M$; (IV) if $A$ is the potential for $F$, its isotropy group Is $(A)$ w.r.t. the gauge transformations is isomorphic to the center of $H_{F}$ on every open neighborhood of $M$.

$H_{F}$ is the holonomy group of the potential $A$. The proof of this theorem will be divided into several steps. Let us first be given a Lie algebra valued 2-form on $M$,

$$
k: T . M \times T . M \mapsto L(G) .
$$

If $E$ is the representation space for $G$ and $L(G), k$ can be identified with the linear operator (which we denote by the same letter)

$$
k: T . M \otimes E \mapsto T . M \otimes E
$$

(the tensor product is taken over the scalars of $E ; T . M$ is the tangent bundle of $M$ and $T \cdot M$ is its cotangent bundle). In a local coordinate system we have a $(\mu A \times \nu B)$ matrix

$$
k=\left(k_{\mu \nu}{ }^{A B}\right)(x),
$$

for $x \in M$, where Greek indices run over spacetime coordinates and the Latin ones denote components in $E$.

Every gauge field $F$ can be seen as one such map. In particular, if we are given the adjoint representation of $L(G)$,

$$
\text { Ad : } L(G) \mapsto L(G) / Z L(G),
$$

where $Z L(G)$ is the center of the algebra, under the above identification

$$
F \mapsto \mathscr{F}=\operatorname{Ad}(F),
$$

where $\mathscr{F}$ is seen as the map

$$
\mathscr{F}: T . M \otimes L(G) \mapsto T . M \otimes L(G) .
$$

For any linear nondegenerate transformation $F \mapsto^{l} F$ one can form similar maps ${ }^{l} F$ and ${ }^{l} \mathscr{F}$.

We finally say that the map $\mathscr{F}$ is algebraically degenerate (or A-degenerate, or a class $I I-$ map $^{1}$ ) if the restricted map

$$
\mathscr{F}(X, Y): L(G) \mapsto L(G)
$$

is degenerate for all $X, Y$, vectorfields on $M$.

We state:

3.6. Proposition. Let $U \subseteq M$ be a nonvoid open set in $M . F$ is ambiguous over $U$ iff $F$ is $A$-degenerate over $U$.

1 The class I maps are those whose degeneracy is a consequence of the fact that $F$ is degenerate as a 2-form over $M$. See [37] for their discussion; they entail the existence of copies which may nowhere be gauge-related 
Proof. As a consequence of Lemma 3.3, $U$ has to contain a nonvoid open set. And nonambiguous $F$ can be uniquely extended to $M-U$.

Now we have:

The condition is necessary [20]. Suppose that $F$ is smooth and can be obtained over $U$ out of two different potentials $A_{1}$ and $A_{2}$. Then $F$ will satisfy two noncoincident differential Bianchi identities (2.11) or (2.15). If we endow $M$ with a metric tensor so that indices can be moved up and down and if (in local coordinates) we write

$$
* F^{\mu \nu}=(1 / 2) \sqrt{|g|} \varepsilon^{\mu \nu \varrho \sigma} F_{\varrho \sigma}
$$

(where $g=\operatorname{det} g_{\mu v}$, the determinant of the metric tensor), the differential Bianchi identities become:

$$
* F_{; \mu}^{\mu v}+\left[A_{\mu},{ }^{*} F^{\mu v}\right]_{-}=0 .
$$

[the (;) denotes covariant derivation w.r.t. the auxiliary metric tensor]. Now write

$$
A_{2}=A_{1}+\varrho
$$

and substitute into (3.2). If we take the difference of the two equations we get:

$$
\left[{ }^{*} F^{\mu v}, \varrho_{v}\right]_{-}=0
$$

which can abbreviated as a matrix equation,

$$
* \mathscr{F} \varrho=0,
$$

or

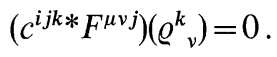

Since $\varrho \neq 0$ on $U, * \mathscr{F}$ is degenerate, that is, $\operatorname{det}^{*} \mathscr{F}=0$ on $U$. And $* \mathscr{F}$ is degenerate if $\mathscr{F}$ is degenerate. We now notice that $\varrho$ takes values in $L(G)$. Since $F$ is supposed not to be degenerate as a 2 -form over $M$, for every $x \in U$, $\mathscr{F}$ kills at least one element in the Lie algebra. Actually as a consequence of (3.3) or (3.4), together with the Jacobi identity, the set of all $\varrho$ that satisfy those relations form a Lie subalgebra of $L(G)$ at every $x \in U$. Thus for all vectorfields $X, Y, \mathscr{F}(X, Y)$ is degenerate, and then $\mathscr{F}$ is $A$-degenerate.

Thus the necessity: if $F$ is ambiguous over $U, \mathscr{F}$ is $A$-degenerate over $U$, provided that it is not degenerate as a 2 -form over $M$.

The condition is sufficient: We will show that if $F$ is $A$-degenerate over $U$, the field $F$ has two different smooth potentials; the ambiguity domain is precisely $U$.

We first notice that as a consequence of the isomorphism

$$
\imath: T . \sigma(U) \mapsto \mathscr{H} \mid U
$$

described in Remark 2.7, one can see the fields $F$ at the point $x \in M$ as being (up to a linear isomorphism) the restriction of the curvature form to the horizontal space at $p \in \pi^{-1}(x)$, that is, for $X^{h}, Y^{h} \in \mathscr{H} \mid p$ one has

$$
F\left(X_{d}, Y_{d}\right)=2 p \varphi\left(X^{h}, Y^{h}\right) p^{-1}
$$


We then apply the Ambrose-Singer theorem [21]:

3.7. Proposition. The $\varphi\left(X^{h}, Y^{h}\right) \mid p$, for all $p \in P$, generate the holonomy algebra $L\left(H_{F}\right)$.

The holonomy algebra is the Lie algebra of $H_{F}$. Before going on with our reasoning we make two assumptions that will be lifted at the end of our proof.

First, we suppose that $\mathscr{F}$ has constant rank on $U$.

Second, we suppose that the bundle is trivial over $U$.

Under the preceding hypotheses, we will need:

\subsection{Proposition. If $\mathscr{F}$ is $A$-degenerate on $U$ then $F$ is reducible on $U$.}

Proof. $G$ is identified with a $n \times n$ matrix group. Let us make the embedding $G \mapsto \mathrm{GL}(n, C)$ and the corresponding embedding $L(G) \mapsto \mathbb{C}(n)$. At a point $x_{0} \in U$, since $\mathscr{F}$ is $A$-degenerate, it takes values inside an ideal

$$
I[\operatorname{Ad} L(G)]\left|x_{0} \subset \operatorname{Ad} L(G)\right| x_{0} .
$$

Thus $F$ takes values inside an ideal

$$
I[L(G)]\left|x_{0} \subset L(G)\right| x_{0} .
$$

The action of $\mathscr{F}$ on $L(G)(U)$ induces a decomposition

$$
L(G)(x)=M(x) \oplus N(x)
$$

on $U$, where $\mathscr{F} N=0$. Let us now endow $L(G)$ with its Cartan metric. Choose a smooth orthonormal basis $[E(x)]$ for $L(G)$ over $U$ such that this decomposition is preserved, that is, such that

$$
\mathscr{F}[E] \mid N(U)=0
$$

This basis exists because the bundle is supposed to be trivial over $U$; as a consequence of the second hypothesis above, $\operatorname{dim} M$ is constant over $U$. Now define a constant basis for $L(G)$ on $U$ as follows : $\left[E_{0}\right](x)=\left(x,[E]\left(x_{0}\right)\right)$. Then there is a unique (up to a constant) gauge transformation $u: U \mapsto \operatorname{GL}(n, \mathbb{C})$ such that $\left[E_{0}\right]=\operatorname{Ad}\left(u^{-1}\right)[E]$. Smoothness in $[E]$ and $\left[E_{0}\right]$ entails the smoothness of $u$.

We now show that $u$ takes values in $G$. In order to do so, we first suppose that $G$ is simple. It is then enough to show that the orbit $\mathcal{O}(K)$ of a fixed element $K$ in the Lie algebra under the adjoint action of $G$ spans the whole Lie algebra. As a consequence every ideal $\mathscr{F}[E]$ can be reached from an isomorphic fixed ideal in $\left[E_{0}\right]$ under the adjoint action of $G$, and thus $F$ can be made to take values inside this fixed constant ideal. Under the action of $G$, the Lie algebra splits as

$$
L(G)=[\mathcal{O}(E)] \oplus S,
$$

where $[$ ] denotes linear closure, and $S$ is a subspace in the Lie algebra which is not reached by the action of $G$. Then there exist $v_{i} \in \operatorname{GL}(n, \mathbb{C}), v_{i} \notin G$, such that $S$ is spanned by the $\operatorname{Ad}\left(v_{i}^{-1}\right)(K)$. By exponentiation one concludes that

$$
\operatorname{Ad}\left(v_{i}^{-1}\right) e^{K} \in G
$$

which is a contradiction. Thus $S=0$ and $\mathcal{O}(K)=L(G)$. 
We have shown that there is a gauge transformation $u: U \mapsto G$ that sends an $A$-degenerate $F$ over an $F^{\prime}$ that takes values inside a fixed ideal in the Lie algebra. We now "lift" our field to the bundle as follows: let $\sigma_{0}=(U, 1)$ be the constant identity cross-section over $U$ (we remember that the bundle is supposed to be trivial over $U$ ). If $F^{\prime}$ takes values inside a fixed ideal in $L(G)$, we attach it to $\sigma_{0}$ as follows: we define

$$
F^{\prime}=2 \varphi \mid \sigma_{0}
$$

[see Eq. (2.9)]. The adjoint action of $G$ concludes the construction of the curvature. It is immediate that $\varphi$ takes values inside a nontrivial ideal of $L(G)$, and as consequence of the Ambrose-Singer theorem, the bundle is reducible.

We must now lift the two restrictions that were imposed during this proof: the triviality of the bundle over $U$ and the simplicity of $G$. If $U$ does not trivialize $P$, we cover $U$ with a collection $V_{i}$ which trivializes the bundle and proceed as above for each $V_{i}$. The bundle local gauge transformations induce the smooth construction of $\varphi$ over the whole $P$.

If $G$ is not simple, we verify that $F$ will take values inside a fixed ideal of $L(G)$ provided that it doesn't vanish on $U$. We can thus apply to each simple ideal in $L(G)$ the previous reasoning. If $F$ vanishes somewhere in $U$, we can partition $U$ into domains where the curvature is not vanishing and patch up the local results, as in the previous paragraph.

We have thus shown that if $\mathscr{F}$ is $A$-degenerate, $F$ is reducible.

In what follows we suppose that this reduction has been already made. We also admit the assumptions made before Proposition 3.8.

We can now conclude the proof of the sufficiency part in Proposition 3.6. As a result of Proposition 3.7, there is a gauge (represented above by the $\sigma_{0 i}$ ) where the elements of the nullspace of $\mathscr{F}$ commute with the holonomy algebra at each point in $U$. We again admit the hypotheses stated before Proposition 3.8.

That is, at each point $U$ we have a decomposition

$$
L(G)\left|x=L\left(H_{F}\right)\right| x \oplus N \mid x .
$$

This decomposition can be continuously and differentiably extended over $U$, that is, one has

$$
L(G)(U)=L\left(H_{F}\right)(U) \oplus N(U),
$$

due to the differentiability of the gauge field $F$.

Let us have a cross-section $\mu: U \mapsto N(U)$. Since $F$ is a gauge field, it is derived from a potential $A$. We suppose that this potential takes values inside the algebra $L\left(H_{F}\right)$; this is the same as requiring that $A$ be the (unique) smallest dimensional solution of the algebraic equations

$$
F_{; \mu}^{\mu v}=* \mathscr{F}^{\mu v} A_{\mu} .
$$

Let now $V \subset U$ be a compact nonvoid set. Define a "bump" function $k: M \mapsto \mathbb{R}$ which is smooth everywhere, vanishes on $M-U$ and is one on $V$. The potential

$$
A^{\prime}=A+\exp (-k \mu) d \exp k \mu
$$


is smooth on $M$, differs from $A$ on $U$, and has $F$ as its field. $A^{\prime}$ is smooth by construction, and has the same field as $A$, for the difference

$$
\begin{aligned}
F(A)-F\left(A^{\prime}\right) & =d \varrho+[\varrho, \varrho]_{-}+2[A, \varrho]_{-}, \\
\varrho & =e^{-k \mu} d e^{k \mu} .
\end{aligned}
$$

$A$ commutes with $\varrho$, and $\varrho$ is a pure-gauge potential, so that (3.7) is zero.

We can now lift the restrictive assumptions that were made during our proof. In order to get a more convenient form for the Bianchi identities we introduced a nondegenerate metric tensor on $M$. It is however immediately clear that the structure of the null space $N(U)$ of $\mathscr{F}$ does not depend on the metric that we are using, provided that this metric be nondegenerate.

We also supposed that $P_{U}$ is trivial. Suppose now it is not. Let $\left[V_{i}\right]$ be a trivializing covering for $U$. We notice that the transformations that glue together the $V_{i} \times G$ have values in the holonomy group $H_{F} \mid U$. Now let the function $\mu: U \mapsto N(U)$ be smooth. This $\mu$ is a cross-section, since it commutes with $H_{F} \mid U$. For a compact $V \subset U$ we define a bump smooth function as above (zero on $M-U$, 1 on $V$ and smooth in between). Then again

$$
\varrho=e^{-k \mu} d e^{k \mu}
$$

is smooth, and $A^{\prime}=A+\varrho$ is a potential for the field $F(A)$ which differs from $A$ on $U$ and which is of class $C^{\infty}$ on $M$.

We finally asked that $H_{F} \mid U$ be of constant dimension on $U$. If not, let us divide $U$ into a collection of sets $\left[W_{i}\right]$ which satisfy:

(I) $\bigcup W_{i}=U$ and each $W_{i}$ is connected;

(II) the dimension of $H_{F}$ is constant on each $W_{i}$;

(III) let $[p]$ be the subset of the integers that contains all values of the dimension of $H_{F}$ on $U$. Then $\bigcup W_{i}$ such that $\operatorname{dim} H_{F} \leqq p, p \in[p]$, is open on $U$.

The last condition means that we extend $H_{F}$ to the boundary with a domain where its dimension drops. In order to get a smooth potential we construct the smooth bump functions $k_{i}: M \mapsto \mathbb{R}$ which are zero on $M-\operatorname{interior}\left(W_{i}\right)$, one on a compact $V_{i} \subset W_{i}$ and smooth otherwise, and use these functions to glue together in a smooth way the local cross-sections

$$
\mu_{i}: W_{i} \mapsto N\left(W_{i}\right) .
$$

We then get a potential that satisfies the desired requirements.

We have thus showed that it is sufficient to have $\mathscr{F}$ degenerate on an open $U \subseteq M$ in order to have a smooth potential ambiguity for $F$. We thus conclude the proof of Proposition 3.6.

Observe that $A$ and $A^{\prime}$ are gauge-connected. $u$ is smooth on the whole of $M$ (it is actually 1 on $M-U$ and nontrivial only on $U$ ). And we notice that

$$
A^{\prime}-A=u^{-1} d u=u^{-1} D(A) u
$$

since by construction $u$ commutes with $A$.

The ambiguous potentials which are not (globally) gauge equivalent among themselves are also to be found with a piece in the null space $N(U)$ of $F$. What really goes on is shown in the next example: 
3.9. Example. Let $F$ be a field which is ambiguous over $U$; we suppose its closure to be compact. Let $A$ be its $L\left(H_{F}\right)$-valued potential and let $N(U)$ be the null space of $\mathscr{F}$. We suppose here that the de Rham group $H^{1}(U, R) \neq 0$.

Consider a nontrivial $N(U)$-valued 1-cocycle $w$ which has the closure of $U$ as its support and is of class $C^{\infty}$. If we cover $U$ with overlapping Poincaré domains (that is, domains where the cocycle $w$ is exact), we can form the local potentials

for $w$. Then while the

$$
\mu_{i}(x)=\int_{0}^{1} w_{\mu}(t x) x^{\mu} d t
$$

$$
u_{i}(x)=e^{\mu_{i}(x)}
$$

cannot be continuously extended to the whole manifold (and even to the whole $U$ ), for all $i$ one has

$$
u_{i}^{-1} d u_{i}=w .
$$

Then $A^{\prime}=A+w$ is a potential for $F$. It is not in general globally gauge-equivalent to $A$. A global gauge transformation must be continuous, and the discontinuities in the several $\mu_{i}$ cannot in general be eliminated with the help of a gauge transformation (see Ezawa and Tze [22]). If the gauge group $G$ is simply connected, one can always manage to find a global gauge transformation that closes the gaps in the potentials for $w$.

3.10. Corollary. $F$ has a single potential on every open $U$ in $M$ iff $F$ is non-degenerate almost everywhere on $M$.

Corollary 3.10 and Definition 3.4 imply:

3.11. Corollary. $F$ has a single potential on every open neighborhood in $M$ iff $F$ is irreducible.

This deals with condition (II) in Theorem 3.5. Also,

3.12. Corollary. $F$ has a single potential on every open neighborhood in $M$ iff the centralizer of $H_{F}$ in $G, C_{G}\left(H_{F}\right)$, coincides with the center of $H_{F}$ on every open neighborhood in $M$.

Proof. Suppose that $F$ has a potential ambiguity on a nonvoid open $U \subset M$. Then on $U$ the proof of Proposition 3.6 shows that one can construct non-central elements in $G$ which commute with $H_{F}$. The converse is immediate.

This deals with condition (III) in Theorem 3.5. We now use a lemma by Singer [23]:

3.13. Lemma. For a potential $A$ under the action of the group of gauge transformations $\mathscr{G}$, its stability group $I s(A)=C_{G}\left(H_{F}\right)$, where $F$ is the field associated to A.

With the help of the lemma above one can prove:

3.14. Corollary. $F$ has a single potential on every open $U \subset M$ iff $I s(A)=Z H_{F}$, where $\mathrm{ZH}_{\mathrm{F}}$ is the center of the holonomy group.

We thus prove (IV) in Theorem 3.5 and conclude its proof. 
3.15. Remarks. (I) For any principal bundle there are always irreducible connections, provided that the fiber group be connected. This is a consequence of the fact that one can always construct a connection on a principal bundle with any finite-dimensional connected Lie group $G$ as its fiber where the holonomy group coincides with $G$, provided that the base space has dimension $\geqq 2$. For its proof see [24].

(II) If all objects are analytic, there are only global copies and $\operatorname{dim} H_{F}$ is constant over $M$.

\section{b) Equivalence Classes of Copies}

We now consider the problem of finding gauge-equivalent and non-gaugeequivalent potentials for the same field. That is, we want to characterize and classify the quotient of all potentials $[A]$ for the field $F$ by the group of gauge transformations $\mathscr{G}$. We exclude potentials that can be restricted to lowerdimensional submanifolds of $M$, or equivalently, we exclude fields that are degenerate as 2-forms over $M$ [25]. Our analysis is a consequence of the results obtained in the preceding part of this section.

We will proceed in two steps. We first state:

3.16. Hypothesis. We suppose that $H_{F}$ is semi-simple and that $L\left(H_{F}\right)$ has dimension greater than 1 w.r.t. its scalars.

We will lift this restriction at the end of the present section.

3.17. Proposition. Let $F$ be copied over a nonvoid open $U \subset M$ and let $H_{F} \mid U$ satisfy Hypothesis 3.16 above. Given a covering $\left[W_{i}\right]$ for $U$ that satisfies conditions (I)-(III) at the end of the proof of Proposition 3.7. Then if $A$ is the $L\left(H_{F}\right)$-valued potential for $F$, its orbit under the action of $\mathscr{G} \mid U$ is given by

$$
\begin{gathered}
A+u^{-1} d u, \\
u^{-1} d u=v^{-1} d \sigma v+v^{-1} d v, \\
d \sigma=\sum d \sigma_{i},
\end{gathered}
$$

where

$$
\sigma_{i} \in \Sigma\left(W_{i}, N\left(W_{i}\right)\right),
$$

this last object being the space of $N$-valued functions of class $C^{\infty}$ whose support is the closure of $W_{i}$, and with $v \in \mathscr{G} \mid U$.

Proof. We first suppose that $\operatorname{dim} H_{F}$ is constant on $U$. Let $A^{\prime}$ be a potential for $F$ which is gauge-related to $A$. Then there exists an $u \in \mathscr{G}$ which is the identity on $M-U$ such that

$$
A^{\prime}-A=u^{-1} D(A) u=u^{-1} d u .
$$

The last equality comes from the fact that $A^{\prime}=A+\varrho$, and

$$
[\varrho, A]_{-}=0 .
$$

Since $A$ is $L\left(H_{F}\right)$-valued, $A$ commutes with $A^{\prime}$ by construction. Equation (3.7) is then reduced to

$$
d \varrho+[\varrho, \varrho]_{-}=0 .
$$


The general solution for this equation has the form

$$
\varrho=u^{-1} d u=v^{-1} d \sigma v+v^{-1} d v
$$

(see below Lemma 3.18), where $v \in \mathscr{G}$ and $\sigma$ is a $N$-valued smooth function that has the closure of $U$ as its support (we observe that $u$ must be a global gauge transformation). The extension to the situation where $\operatorname{dim} H_{F}$ is not constant on $U$ can be done as in the proof of Proposition 3.6.

We now state in full detail the step represented by Eq. (3.18) in the preceding proof, since it has direct bearing on several aspects of the copy problem:

3.18. Lemma. If $\varrho$ is a smooth solution over $U \subset M$ for the equation

$$
D(\varrho) \varrho=d \varrho+[\varrho, \varrho]_{-}=0,
$$

then on $U, \varrho \in(\mathscr{G} \mid U)(\sigma)$, where one can write

$$
\sigma=\sigma^{i}(x) X^{i}
$$

$\mathscr{G} \mid U(\cdot)$ denoting the orbit of an object under the action of the restricted group $\mathscr{G} \mid U$; the $X^{i}$ are a basis for the algebra $L(H)$ where $\varrho$ has values and each $\sigma^{i}(x)$ is a representative of a cohomology class in the de Rham group $H^{1}(U, \mathbb{R})$.

Proof. Let $U$ be covered by a collection $B_{k}$ of open balls. As $P \mid B_{k}$ is trivial, $\mathscr{G} \mid B_{k}$ is given by

$$
\mathscr{G} \mid B_{k}=\left\{u \mid u: B_{k} \mapsto H\right\},
$$

(smooth maps!). Consider the restriction $D(\varrho) \varrho=0 \mid B_{k} . \varrho=0$ and $\left(\mathscr{G} \mid B_{k}\right)(0)=u^{-1} d u$, $u \in \mathscr{G} \mid B_{k}$ are solutions for this restricted equation. There are no other solutions, for suppose that

$$
\theta \neq u^{-1} d u
$$

for $u \in \mathscr{G} \mid B_{k}$. Then $\theta$ takes values in a nonzero subalgebra of $L(H)$. But as

$$
D(\varrho) \varrho=0
$$

the holonomy algebra is 0 . Then $\theta$ cannot be a solution for (3.19) on $B_{k}$.

Also $\varrho$ is either exact or gauge-equivalent to an exact 1 -form over $B_{k}$. For any $u \in \mathscr{G} \mid B_{k}$ can be written as a product

$$
u=\prod_{j} e^{\mu_{j}(x)},
$$

or

If one writes this as

$$
u=e^{\mu_{1}(x)} \prod_{j} e^{\mu_{j}(x)}, \quad j \neq 1
$$

$$
u=e^{\mu} v
$$

one has

$$
\varrho(x)=u^{-1} d u=v^{-1} d \mu v+v^{-1} d v .
$$

Naturally, the

$$
\mu_{j}: B_{m} \mapsto L(H),
$$


are smooth. It is also straightforward to check that $\varrho$ will not in general be exact. Thus

$$
\varrho(x) \in\left(\mathscr{G} \mid B_{k}\right)(d \mu)
$$

for all

$$
\mu: B_{m} \mapsto L(H)
$$

of class $C^{\infty}$ on $B_{m}$.

In order to extend these piecewise solutions to the whole of $U$ so that $\varrho$ stays smooth on $U$, one extends $v$ to the whole of $U$ and picks the several $d \mu$ over the $B_{m}$ to be local descriptions for a nontrivial de Rham 1-cocycle $\theta$ over $U$,

We thus conclude the lemma.

$$
\theta=\theta^{i} X^{i}
$$

With its notation together with the condition that the closedness of $\theta$ means the closedness of each component $\theta_{i}$, we have:

3.19. Corollary. If $\theta$ is closed,

$$
u^{-1} d u=\theta \mid B_{m}
$$

has as solution

$$
u(x)=\exp X^{i} \int_{0}^{1} \theta_{\mu}^{i}(t x) x^{\mu} d t
$$

Proof. By the Poincaré lemma,

$$
\mu^{i}(x)=\int_{0}^{1} \theta_{\mu}^{i}(t x) x^{\mu} d t .
$$

3.20. Corollary. If $\exp : L(H) \mapsto H$ is onto, every $\varrho$ which is a solution for (3.19) is of the form

where each $\theta^{i}$ is a 1-cocycle on $U$.

$$
\theta=\theta^{i} X^{i}
$$

Proof. In that case every $u \mid B_{k}=e^{\mu_{j}}$.

3.21. Remarks. (I) We notice that while $u(x)$ in the preceding results is not in general continuous over the whole subset $U \subset M, \varrho=u^{-1} d u$ is continuous provided that it be gauge-equivalent to a linear combination of 1-cocycles. This is the main obstruction to the construction of global gauge mappings that send the several copied continuous potentials for $F$ over each other. It would be easy to construct noncontinuous potentials for a field which is copied over $U$; these potentials would not be gauge equivalent to a continuous potential, since in this case Eq. (3.15) would never give a global gauge transformation due to the discontinuity in $\varrho$.

(II) If $\theta$ is a linear combination of cocycles,

$$
\begin{gathered}
\lambda u^{-1} d u=\theta \mid B_{k}, \\
\lambda \neq 0
\end{gathered}
$$


( $\lambda$ a constant) has as its solution

$$
u(x)=\exp (1 / \lambda) X^{i} \int_{0}^{1} \theta_{\mu}^{i}(t x) x^{\mu} d t .
$$

We now define $H$ to be the smallest Lie subgroup of $G$ that contains both $H_{F}$ and $C_{G}\left(H_{F}\right)$. It can be checked that $H=\left\{h z \mid h \in H_{F}, z \in C_{G}\left(H_{F}\right)\right\}$. We also denote by [A] the set of all potentials for a copied field $F=F(A)$; their gauge-equivalence classes are denoted by $[A] / \mathscr{G}$ (or by a convenient restriction).

We can state:

3.22. Proposition. Let the conditions be as in (3.17). We also suppose that $\operatorname{dim} H_{F}$ is constant on $U$. The following conditions are exclusive: (I) Either the first de Rham group $H^{1}(U, \mathbb{R})=0$ and every smooth potential for $F$ belongs to the orbit $\mathcal{O}_{0}(A)$ of the $L\left(H_{F}\right)$-valued potential for $F$ (that is, every potential is gauge-equivalent to $A$ ), or (II) $H^{1}(U, \mathbb{R}) \neq 0$ and there is an isomorphism

$$
\imath:[A] / \mathscr{G} \mid U \mapsto \pi_{1}(G, H, 1)
$$

between the set of gauge-equivalence classes of potentials over $U$ for $F$ and the first relative homotopy group $[26] \pi_{1}(G, H, 1)$.

Proof. If $H^{1}(U, \mathbb{R})=0$, there are only trivial cocycles on $U$ and (3.17) has as its unique solutions those described in Proposition 3.17. That is, every continuous potential for $F$ is gauge-equivalent to $A$ over $U$.

Suppose now that $H^{1}(U, \mathbb{R}) \neq 0$. We then have nontrivial cocycles. We classify the potentials associated to the different cohomology classes using a technique described in detail by Ezawa and Tze [27]. Let $S^{1}$ be a 1-cycle in $U$ homeomorphic to the 1-sphere such that for a nontrivial Lie algebra valued cocycle $\varrho$ on $U$,

$$
\int_{S^{1}} \varrho \neq 0 \text {. }
$$

Choose a fixed point $x_{0} \in S^{1}$ and define the map

$$
S^{1} \mapsto g\left(x_{0}, S^{1}\right)=\exp \int_{S^{1}} A^{\prime}
$$

from the space $S^{1}\left(x_{0}, U\right)$ of cycles homeomorphic to the 1-sphere in $U$ which contain $x_{0}$ to the loops in $G$ which start from 1 (image of $x_{0}$ ) and have its endpoint in the group $H$, for (3.27) equals

$$
\exp \int_{S^{1}}(A+\varrho)=\left(\exp \int_{S^{1}} A\right)\left(\exp \int_{S^{1}} \varrho\right)
$$

$A+\varrho=A^{\prime}$. The equivalence classes of such loops are the elements of $\pi_{1}(G, H, 1)$.

3.23. Remark. $\mathcal{O}_{0}(A)$ is sent over the neutral element in $\pi_{1}(G, H, 1)$.

Let conditions be as in Proposition 3.22, but for the following: if $V \subset U$ is a nonvoid open set, let us write $U=V \cup W$. We suppose that $\operatorname{dim} H_{F}\left|V<\operatorname{dim} H_{F}\right| W$, and also that $W$ is connected. Then 
3.24. Proposition. The equivalence classes of smooth potentials for $F$ w.r.t. the action of $\mathscr{G}_{U}$ are given by:

$$
\pi_{1}(G, H \mid V, 1) \times \pi_{1}(G, H \mid W, 1) .
$$

Proof. Fix one potential $A$ for $F$ over $U$. This potential becomes ambiguous over $V$, and is classified according to Proposition 3.22. We now allow $A$ to vary over all potentials for $F$ on $W$, and get (3.29).

If $A^{1}$ and $A^{2}$ are two potentials for $F$ which differ only over $V$ and which are smooth, the gauge transformation (3.23) can be smoothly extended to the whole $U$ whenever (I) $w=A^{1}-A^{2}$ is exact and (II) $W$ is connected. Suppose that $V$ can be covered by a single coordinate patch $C, V \subset C$. If we place the origin $0 \in C-V$, outside $V$ the transformation (3.23) reduces to the identity. As $W$ is connected, and as everything is smooth, we get the desired extension. If $V$ is not contained in a single coordinate patch, we cover it with a family of coordinate patches and proceed as above. Outside $V$ the potential for $w$ becomes zero, and again by continuity we get our result.

If we allow the dimension of $H_{F}$ to vary over $U$ we may meet an obstruction when extending a gauge transformation between domains with different holonomy groups. This obstruction occurs if $U-V$ is not connected.

We see that the only necessarily nonvoid class in $[A] / \mathscr{G} \mid U$ is the orbit $\mathcal{O}_{0}(A)$. We also see that the breakdown of gauge equivalence between the elements of $[A] / \mathscr{G}_{U}$ is a consequence of the nontrivial connectivity of the region $U$ (or of the region $U-\bigcup V$, if the dimension of the holonomy group varies over $U$ ). Since each cocycle $\varrho$ is supposed to be smooth and of support $\operatorname{cl} U$, we notice that the corresponding potentials $A+\varrho$ [where $A$ is $L\left(H_{F}\right)$-valued] are continuous over $M$. This is in strong contrast with the situation in the abelian case, where as a consequence of the existence of a nontrivial second de Rham group $H^{2}(M, \mathbb{R})$ we have discontinuous potentials for a continuous field [28]. We also notice that for an adequately small $U$ one can put $H^{1}(U, \mathbb{R})=0$, and as a result obtain local gauge transformations that make all connections equivalent. But we cannot always extend these results to a larger $V$ that includes this $U$ due to the obstructions discussed above. For an analysis of the case of varying holonomy dimension along different lines see Mostow's paper [19].

A nontrivial $H^{1}(U, \mathbb{R})$ is only a necessary condition for the existence of nonequivalent classes of copies. One can assert:

3.25. Proposition. $[A]=\mathcal{O}_{0}(A)$ iff $\pi_{1}(G)=0$. We suppose valid the conditions of Proposition 3.22.

Proof. Consider the exact sequence

$$
\pi_{1}(H, 1) \rightarrow \pi_{1}(G, 1) \rightarrow \pi_{1}(G, H, 1) \rightarrow \pi_{0}(H, 1)
$$

[29]. Its exactness implies the proposition. We observe that $H_{F}$ is connected as a consequence of Remark 3.15(I).

For example, if $G$ is special unitary, there are no nontrivial potentials for a reducible field. 
3.26. Proposition. Conditions are as in Proposition 3.22. If $H$ is contained in a simply-connected domain of $G$, the fundamental group $\pi_{1}$ classifies the equivalence classes $[A] / \mathscr{G}_{U}$.

Proof. Also a consequence of (3.30). See [30].

We can now lift the restriction imposed by Hypothesis 3.16. We admit that $H_{F}$ may contain a nontrivial abelian subgroup [or that it is isomorphic to $U(1)$ or to $\mathbb{R}]$. But this does not change the structure of the subgroup $H$ in an essential way, and so the preceding results remain valid. One can however consider a particular situation which has bearing on an important class of examples of the copy phenomenon $^{2}[31]$ :

3.27. Proposition. Let $X^{i}$ be a basis for $L\left(H_{F}\right)$. Given a nonzero $F=F^{i} X^{i}$, if for all $i$, $d F^{i}=0$ on an open $U \subseteq M$, then $F$ is copied over $U$.

Proof. Consider the Bianchi identity (3.2). Since $d F^{i}=0$, one has

$$
* \mathscr{F} A=0 \text {. }
$$

As $F$ is nonzero, so is $A$. As a consequence, $* \mathscr{F}$ is degenerate and by $3.6 F$ is copied over $U$.

We can also state:

3.28. Proposition. Let $F=[u, v]_{-}$, where $u$, $v$ are $L(G)$-valued 1 -forms on $M$. Let also $d F^{i}=0$ on $U$ for all $i$. Then, for $u=u^{i} X^{i}$ and $v=v^{i} X^{i}, d u^{i}=d v^{i}=0$, for all $i$.

Proof. In a particular Lie algebra basis one has

$$
F^{i}=c^{i j k} u^{j} \wedge v^{k} \text {. }
$$

Since the cocycles form an algebra which is closed under the exterior product, $d F=0$ implies that $d u=d v=0$.

3.29. Proposition (Bollini et al. [32]). Let $G=\mathrm{SU}(2)$ or $\mathrm{SO}(3, \mathbb{R})$. If $d F=0$ then $F$ generates a $U(1)$ subgroup of $\mathrm{SU}(2)$ or an $\mathrm{SO}(2, \mathbb{R})$ subgroup of $\mathrm{SO}(3, \mathbb{R})$.

Proof. A direct consequence of Proposition 3.27.

3.30. Remark. The original example given by $\mathrm{Wu}$ and Yang belongs to this category, with $G=\mathrm{SO}(3, \mathbb{R})$ and $M=S^{1} \times S^{2}$. The nontrivial geometry of the base manifold is hidden in the characterization given by Wu and Yang. As we are given a single potential for a monopole field, there is a Dirac string; the monopole can be visualized as the source for lines of force that go to infinity and come back through the string. If we compactify $\mathbb{R}^{3}$, cut out a small 2-sphere round the monopole, another one around the $\infty$-point and identify both, we get a manifold which is homeomorphic to $S^{1} \times S^{2}$. For details and calculations see the Bollini, Giambiagi, and Tiomno paper. We notice that in the Wu-Yang example there is a whole family of potentials that are nowhere equivalent to the monopole potential; these potentials exist even if we are given a trivial bundle over $\mathbb{R}^{3}$. For their discussion see [37].

2 For a discussion of the other aspects of this particular class of examples, see [37], and Solomon's paper in $[1]$ 
Before we conclude it is interesting to notice that the classifying group in Proposition 3.22 is the same used by Ezawa and Tze [33] to classify NielsenOlesen vortices on a spacetime manifold. Their discussion is easily translated into our language.

\section{Conclusions}

Gauge field copies exist here whenever the holonomy group of a connection is smaller than the whole gauge group, which is the fiber group of the principal bundle where we construct our gauge fields. The preceding analysis and in particular Eq. (3.28) suggest that these copied fields may play a role similar to that of the electromagnetic potential in the Aharonov-Bohm experiment [34]. Also since nontrivial cocycles on manifolds without boundary can be associated [35] to point sources which are outside the manifold, some kind of monopole-like picture could perhaps be given to the non (globally) gauge equivalent connections for a copied field. And finally there is a direct relationship between the copy phenomenon and symmetry breaking in nonabelian gauge fields; one example of such a relationship is given by the Ezawa and Tze classification for the Nielsen-Olesen vortices. It can be also shown that the $A$-degenerate fields which were considered in the present paper possess (locally at least) the Gribov Coulomb gauge degeneracy. For details see [36, 37].

An analysis of the situation of copied fields in the manifold of curvatures and connections for a given principal bundle can show that non-copied fields are precisely the "linearization stable" gauge fields in the sense of Arms [36]. This will be considered in a forthcoming paper.

Acknowledgements. The author thanks C. G. Bollini, and J. J. Giambiagi who introduced him to the copy problem. He also acknowledges interesting discussions with G. G. Emch, J. Harper, and N. Stein, as well as a fruitful conversation with R. Palais.

He finally wishes to thank S. L. Segal and the Department of Mathematics, University of Rochester, for its hospitality.

\section{References}

1. Wu, T.T., Yang, C.N.: Phys. Rev. D 12, 3843-3844 (1975; Gu, C.H., Yang, C.N.: Sci. Sin. 18, 484 (1975); Gu, C.H., Yang, C.N.: Sci. Sin. 20, 48 (1977); Deser, S., Wilczek, F.: Phys. Lett. 65 B, 391-393 (1976); Deser, S., Drechsler, W.: Phys. Lett. 86 B, 189-192 (1979); Roskies, R. : Phys. Rev. D 15, 1731-1732 (1977); Calvo, M.: Phys. Rev. D 15, 1733-1735 (1977); Halpern, M.B.: Phys. Rev. D 16, 1798-1801 (1977); Halpern, M.B.: Nucl. Phys. B 139, 477-489 (1878); Coleman, S.: Phys. Lett. 70 B, 59-60 (1978); Bollini, C.G., Giambiagi, J.J., Tiomno, J.: Preprint A-0022/78. Centro Brasileiro de Pesquisas Fisicas, Rio de Janeiro RJ, Brazil; Bollini, C.G., Giambiagi, J.J., Tiomno, J. : Phys. Lett. 83 B, 185-187 (1979); Solomon, S. : Nucl. Phys. B 147, 174-188 (1979); Mostow, M. : Commun. Math. Phys. 78, 137-150 (1980); Doria, F.A.: On an example of field copies by Deser and Wilczek. Dept. of Mathematics, Univ. of Rochester (1980)

2. Wu, T.T., Yang, C.N.: Phys. Rev. D 12, 3843-3845 (1975)

3. Bollini, C.G., Giambiagi, J.J., Tiomno, J.: Preprint A-0022/78. Centro Brasileiro de Pesquisas Físicas, Rio de Janeiro RJ, Brazil

4. Cho, Y.M.: J. Math. Phys. 16, 2029-2035 (1975)

5. Kobayashı, S., Nomizu, K.: Foundations of differential geometry. I. New York, London: Interscience 1963; Sternberg, S.: Lectures on differential geometry. Englewood Cliffs, N.J.: PrenticeHall 1964 
6. Atiyah, M.F., Jones, J.D.S.: Commun. Math. Phys. 60, 97-118 (1978); Atiyah, M.F.: Geometry of Yang-Mills Fields. Pisa: Scuola Normale Superiore 1979

7. Doria, F.A. : Topologia e campos de gauge. Habilitation thesis (in Portuguese), Instituto de Física, Universidade Federal do Rio de Janeiro, Rio de Janeiro RJ, Brazil

8. See [5]

9. See [5]

10. Wu, T.T., Yang, C.N.: Phys. Rev. D 12, 3845-3857 (1975)

11. Private communication

12. Kobayashi, S., Nomizu, K.: Op. cit., pp. 75-77

13. See [12]

14. Sternberg, S.: [5]

15. Kobayashi, S., Nomizu, K.: Op. cit., Proposition 7.3, 142

16. Daniel, M., Viallet, C.M.: Rev. Mod. Phys. 52, 175-197 (1980)

17. See [3]

18. Deser, S., Drechsler, W.: Phys. Lett. 86 B, 189-192 (1979)

19. Mostow, M.: Commun. Math. Phys. 78, 137-150 (1980)

20. Several authors have already discussed an almost equivalent necessary condition (that turns out to be also a sufficient condition for the existence of copies in the general case, see [37]), the full degeneracy of the matrix * $\mathscr{F}$. See Deser and Wilczek in [1]. See also the papers by Roskies, Calvo, and Halpern in [1]

21. Ambrose, W., Singer, I.M. : Trans. Am. Math. Soc. 75, 428-443 (1953); Kobayashi and Nomizu and Sternberg, op. cit., have also proofs of this theorem

22. Ezawa, Z.F., Tze, H.C.: J. Math. Phys. 17, 2228-2232 (1976)

23. Singer, I.M.: Commun. Math. Phys. 60, 7-12 (1978); Narasimhan, M.S., Ramadas, T.R.: Commun. Math. Phys. 67, 121-136 (1979)

24. Nomizu, K.: Nagoya Math. J. 10, 101-103 (1956)

25. We exclude the cases of connections which are kept invariant when restricted to a bundle over a lower-dimensional submanifold of $M$. In the 3-dimensional case the connection form when extended to a 4-dimensional $M$ may lead to the degeneracy of $* \mathscr{F}$ without however implying the existence of copies over the 3-manifold (see [37]). The 2-dimensional case is unique in that every 2-curvature is copied. See Deser and Wilczek in [1]

26. Hu, S.T.: Homotopy theory, p. 110. London, New York: Academic Press 1959

27. See [22]

28. As a physical interpretation of this fact see Doria and Abrahão: J. Math. Phys. 19, 1650-1653 (1978)

29. $\mathrm{Hu}$, S.T.: [26], p. 115

30. See [22]

31. See [3]

32. See $[3]$

33. See $[22]$

34. Aharonov, Y., Bohm, D.: Phys. Rev. 115, 485-494 (1959)

35. Doria, F.A.: Anais Acad. Bras. Ciências 51, 207-209 (1979) See also [7]

36. Arms, J.M.: J. Math. Phys. 20, 443-453 (1979); Doria, F.A.: Some consequences of the Wu-Yang ambiguity. Preprint. Dept. of Mathematics, Univ. of Rochester (1980)

37. Doria, F.A.: On the existence of the Wu-Yang ambiguity. Anais Acad. Bras. Ciências (to be published); Doria, F.A.: Quasi-abelian and fully non-abelian gauge field copies. Preprint. Dept. of Mathematics, Univ. of Rochester (1980)

Communicated by R. Stora

Received December 26, 1979; in revised form August 21, 1980 